\title{
Lidil
}

Revue de linguistique et de didactique des langues

$30 \mid 2004$

Acquisition et enseignement de la morphographie

\section{Les mots du bled}

Création contemporaine en langues maternelles, les artistes ont la parole

\section{Cyril Trimaille}

\section{(2) OpenEdition}

\section{Journals}

Édition électronique

URL : http://journals.openedition.org/lidil/1043

DOI : $10.4000 /$ /idil. 1043

ISSN : 1960-6052

Éditeur

UGA Éditions/Université Grenoble Alpes

\section{Édition imprimée}

Date de publication : 1 décembre 2004

Pagination : 183-188

ISBN : 2-914176-11-2

ISSN : $1146-6480$

\section{Référence électronique}

Cyril Trimaille, "Les mots du bled », Lidil [En ligne], 30 | 2004, mis en ligne le 29 janvier 2008, consulté le 23 septembre 2020. URL : http://journals.openedition.org/lidil/1043 ; DOI : https://doi.org/10.4000/ lidil.1043 


\section{Note de lecture}

Les mots du bled, Création contemporaine en langues maternelles, les artistes ont la parole.

Entretiens avec Fellag, Cheb Sahraoui, Allalou, Youssef, Fadel, Fadhel Jaïbi, Baâziz, Ben Mohamed, Aziz

Chouaki, Gyps, Amazigh Kateb, Omar Sayed, Rachid Taha, Hamma, présentés et édités par Dominique Caubet, L'Harmattan, Paris, Budapest, Torino, Collection Espaces discursifs, 239 p.

Dominique Caubet est (socio)linguiste, professeur d'arabe maghrébin à l'INALCO (《Langues $O$ '») et dirige le CREAM, Centre de recherche et d'études sur l'arabe maghrébin. Parmi ses domaines de compétence et de prédilection, la description de l'arabe marocain et plus largement maghrébin (Négation en berbère et en arabe maghrébin, avec S. Chaker, La codification des langues en France, avec S. Chaker et J. Sibille), elle est spécialiste des situations de plurilinguisme au Maghreb et en France et s'intéresse à ce titre aux répertoires verbaux plurilingues des jeunes issus de l'immigration maghrébine, et à la circulation d'éléments langagiers entre ici (France) et là-bas (Maghreb) ${ }^{1}$.

Outre une préface de Hadj Miliani, l'ouvrage comporte une introduction, un recueil d'entretiens menés entre 1995

1. Parlers jeunes ici et là-bas. Pratiques et représentations est le titre d'un ouvrage qu'elle a co-édité en 2004 avec J. Billiez, Th. Bulot, I. Léglise et C. Miller, L'Harmattan. 
et 2004 auprès d'artistes maghrébins ${ }^{2}$, qui utilisent le langage et les langues comme matière première de leur travail; une sorte de postface et un glossaire viennent clore le travail.

Dans la partie introductive, $D$. Caubet présente la genèse et les enjeux du séminaire qui s'est prolongé par cette publication. Elle pose d'emblée la nécessité de «s'attaquer à la langue de bois qui n'est jamais bien loin dès que l'on parle d'arabe, en démontant dans les faits quelques idées reçues concernant les langues du Maghreb $»$ (p. 9). Pour ce faire, elle débute son introduction par une contextualisation, donnant ainsi au lecteur d'utiles repères sur la situation sociolinguistique du Maghreb. Elle fait état du foisonnement et de la vitalité des langues du quotidien maghrébin, partagées par la quasi-totalité des locuteurs.

À propos de l'Algérie, D. Caubet ainsi que plusieurs des artistes interviewés pointent, en convoquant notamment des propos de l'écrivain Kateb Yacine, le décalage entre des politiques linguistiques monolingues et le plurilinguisme des peuples. D'ailleurs, la poursuite fantasmatique d'une unité nationale et panarabique, n'est pas sans rappeler, à certains égards, les siècles d'imposition du français dans l'Hexagone, et la portée de cette réflexion saisissante de l'universitaire et dramaturge Aziz Chouaki semble, de fait, pouvoir être étendue à bien d'autres situations sociolinguistiques et éducatives que celle de l'Algérie: "J'ai l'impression que tout ce qu'ont fait les régimes algériens jusqu'à aujourd'hui, c'est justement de briser ce lien naturel, qui existait entre la mère et son enfant pour en instituer un autre qui est virtuel, qui n'a pas de prise dans le petit quotidien simple. A telle enseigne que par exemple, des enfants qui apprennent l'arabe classique à l'école, quand ils rentrent à la maison, corrigent la mère qui se retrouve exclue au profit d'une autre mère, disons supérieure, qui n'existe pas, qui est virtuelle; et qui est en fait pilotée par une espèce de géopolitique en carton-pâte et ça fait des dégâts considérables à tous les niveaux » (p. 156).

2. Les artistes interviewés (Sofiane Faoucène et Nabil Acef) l'ont été dans le cadre d'un séminaire mis en place à l'INALCO en 1995. 
De manière réactive, se sont développés «pour toute une partie de la population [algérienne], artistes y compris, une pratique systématique des mélanges et d'un humour d'autodérision dévastateur (dont Fellag est la figure emblématique) [ainsi qu'une] «haine de soi» souvent accompagnés d'un retour fantasmatique à un passé arabo-musulman mythique, égalitaire et pur qui aboutit, dans ses formes extrêmes, à un repli sur soi et à une violence inouïe» (p. 12). Cette autodévalorisation et la volonté de passer outre voire de la renverser, sont des traits récurrents dans les propos des créateurs interviewés.

Au long des interviews ${ }^{3}$, des chanteurs, poètes, hommes de théâtre ou dessinateur, qui ont pour point commun d'être plurilingues, de vivre et de créer dans leurs «langues maternelles » parlent donc de leurs relations aux langues (et par la force des choses de leur rapport au pouvoir et à la censure), de la place que prennent ces dernières dans leurs activités artistiques, mais aussi de l'importance qu'elles revêtent dans leur parcours. À propos de la désignation de ces langues du quotidien maghrébin (mais aussi français), un des regrets que l'on peut exprimer est que l'auteure ne mette pas à profit cet ouvrage destiné à une diffusion relativement large, et pas uniquement à des spécialistes, pour donner un écho aux questionnements (et remises en question) ${ }^{4}$ qui existent à propos de la notion de langue maternelle qui masque eñ fait des réalités très différentes. Certes, la catégorie est mobilisée par plusieurs artistes, mais il est remarquable que deux des artistes interviewés, Fellag et Aziz Chouaki, considèrent que leur langue maternelle est un «mixte» de trois langues, le berbère, l'arabe algérien et le français... ${ }^{5}$

3. On peut noter que la plupart des interactions étant définies comme bilingues par les protagonistes, des alternances codiques s'y manifestent, que l'auteure a conservées en proposant une traduction.

4. Voir par exemple l'article critique consacré à cette notion de LM dans l'ouvrage collectif dirigé en 1998 par C. Baker et S. Prys Jones, Encyclopedia of Bilingualism and Bilingual Education, Multilingual Matters, p. 47.

5. Rachid Taha considère l'arabe classique et le français comme des langues étrangères, le français étant, selon lui, devenu sa «langue paternelle, la langue du père qui était immigré » (p. 213). 
Mais il n'en reste pas moins qu'en donnant la parole à des artistes qui montrent et célèbrent la vitalité et la richesse des langues «du quotidien» et du plurilinguisme, c'est donc à une véritable entreprise de "politique linguistique par le bas» (Billiez, 1997) et de déminorisation que s'attache D. Caubet. Elle se fait ainsi - non sans une certaine jubilation - l'écho de la subversion des créateurs qui en donnant à voir, à lire ou à entendre des langues sans statut et des mélanges refoulés parce que stigmatisés, légitiment leur existence et transgressent l'ordre monolithique et monoculturel. Si la jubilation du mélange ou de l'alternance est souvent dite et revendiquée, on sent également poindre, en filigrane, les thèmes de la construction de compétences plurilingues et celui des appropriations «non-didactiques» des langues sans statut.

Néanmoins, pour plusieurs créateurs, la darja ne doit pas seulement être perçue comme le vecteur d'une liberté d'expression (re)trouvée: c'est le plus souvent la langue de l'enfance et des affects dans laquelle tout ne peut pas être dit; c'est aussi la langue des «formes traditionnelles de littérature orale, contes, proverbes, énigmes qui véhiculent une morale et qui reconduisent l'ordre ancestral local» (p. 15). Il apparait clairement que pour ces artistes, les tabous et les frontières du dicible sont dépassés grâce au bi-plurilinguisme, et notamment grâce au français, qui selon Aziz Chouaki «n'appartient pas qu'à la France», même si plusieurs des interviewés verbalisent une représentation apparemment répandue en Algérie, selon laquelle un homme qui parle français ferait "efféminé" " (Gyps et Allalou), voire un traitre à la patrie.

La visibilité que confère la création contemporaine aux langues et variétés vernaculaires du Maghreb ne se limite pas à cet espace nord africain. En effet, de nombreux artistes, Fellag, Amazigh Kateb (Gnawa Diffusion), Allalou, Rachid Taha, Baâziz, Sofiane Faoucène et Nabil Acef (rappeurs du groupe Hamma) travaillent aujourd'hui en France, jouant aussi un rôle déterminant dans la mise en visibilité de l'arabe maghrébin et l'embryon de reconnaissance dont il jouit aujourd'hui. Ces contributions nourrissent et vivifient ce que D. Caubet choisit de nommer une «scène culturelle française 
plurielle ${ }^{6}$ » s'inscrivant non pas dans une «double culture» mais dans une culture française renouvelée ${ }^{7}$. Et la circulation des traits culturels et linguistiques ne s'arrête pas là, puisqu'en retour ces artistes ont une audience et une influence artistique « au bled».

Les liens complexes entre langues, biographies, engagements, trajectoires et stratégies identitaires (personnelles, sociales, nationales, artistiques...), apparaissent donc comme une sorte de fil rouge dans les propos des interviewés, qui conçoivent leur activité de création - d'essence fondamentalement langagière - comme une forme ou au moins un lieu de résistance linguistique et sociale. Si l'introduction était essentiellement consacrée à la situation des pays du Maghreb, une partie des discours recueillis et la conclusion de Dominique Caubet sont davantage tournées vers la France. Ils permettent de prendre la mesure de l'évolution qui s'y est opérée depuis le début des années 1980, quand par exemple la reprise de C. Trenet, «Douce France» chantée par le groupe Carte de séjour (avec R. Taha) sonnait encore comme une tentative de renversement sémantique du stigmate, qu'I. Leonetti-Taboada (1991: 64) considère comme le premier signe de prise de conscience d'une assignation d'identité minoritaire, de domination et de discrimination. Mais si évolution il y a eu, le chemin à parcourir vers la conquête de l'égalité des citoyens dans la pluralité linguistique reste encore long et siñueuxux, et̂ néces-

6. D. Caubet en veut pour élément de preuve le grand succès de l'album « 1, 2, 3 soleil », rassemblant Faudel, Rachid Taha et Khaled, et plus particulièrement du titre Ya rayah (repris au répertoire de Dahmane El Harrachi). Elle mentionne aussi le film «La vérité si je mens» (comprenant de nombreux énoncés en arabe marocain) et les spectacles de Jamel Debbouze, dans lesquels la darja est l'objet d'usages sans traduction. On pourrait ajouter à cette liste les nombreux rappeurs dont les textes sont plurilingues et font une place aux variétés d'arabe maghrébin (Fonky Family, 113, Freeman,...).

7. Malgré ces signes encourageants, l'auteure note tout de même que, sans être les seules (elle cite le cas de la liste des Motivés et de quelques hommes et femmes politiques issus de l'immigration), les arts et le sport demeurent deux sphères professionnelles qui offrent une perspective de mobilité sociale ascendante et une visibilité positive des immigrés ou de leurs descendants. 
sitera bien d'autres ouvrages de ce type. À moins que le travail des chercheurs n'ait aucune prise sur les décisions politiques...

Cyril Trimaille

BILLIEZ J. (1997): Bilinguisme, variations, immigration: regards sociolinguistiques, Dossier présenté en vue de l'Habilitation à diriger des recherches, 2 volumes, Université Stendhal-Grenoble 3.

LeONETTI-TABOADA I. (1991): "Stratégies identitaires et minorités », Migrants Formation, n 86, p. 54-73.

De Gaulmyn, M.-M., R. Bouchard \& A. Rabatel (dir.), 2001, Le processus rédactionnel: Écrire à plusieurs voixx, Paris, L'Harmattan, 317 p. ISBN : 2-2475-0883-8.

Cet ouvrage collectif, qui réunit plus d'une vingtaine d'auteurs $^{8}$, traite des interactions dans et par le processus rédactionnel, que ce soit au niveau de la genèse des textes écrits, captée au vif, ou au niveau du méta-texte après coup. Par des voies d'approche variées, les différentes contributions participent chacune à sa manière à une réflexion autour de la rencontre entre oral et écrit, longtemps tenus à distance dans la logique du «grand partage».

Dans l'introduction (p.9-16), les coordinateurs (M.-M. de Gaulmyn, R. Bouchard et A. Rabatel) expliquent le contexte scientifique et intellectuel dans lequel s'insère l'ouvrage, qui prend sa source dans une rencontre interdisciplinaire entre chercheurs en Sciences Humaines (voir aussi de Gaulmyn,

8. Par ordre alphabétique: J. Andriessen, D. Apotheloz, J.-P. Bernié, A.-C. Berthoud, J.-M. Besse, N. Bonnardel, R. Bouchard, C. Brassac, A. Camps, A. Chevalier, P. Coirier, J. David, L. Gajo, M.-M. de Gaulmyn, O. Guasch, J. Miecznikowski-Fünfschilling, M. Milian, L. Mondada, A. Moulin, A. Piolat, S. Plane, A. Rabatel, T. Ribas, J. Vacherand-Revel. 